\title{
Incidence of allergic rhinitis in children with residual snoring and sleep symptoms after adenotonsillectomy
}

Lernik Sarkissian, Lala Sarkissian, Thomas E Stewart, Thomas Guy, Stuart G Mackay

\section{Background and objectives Allergic rhinitis may contribute to sleep disordered breathing (SDB) in children. Although adenotonsillectomy is commonly performed to treat SDB, some patients will return to their primary practitioners with residual sleep symptoms. The aim of this study was to assess the incidence of allergic rhinitis via radioallergosorbent testing (RAST) in children undergoing adenotonsillectomy who had residual snoring or sleep symptoms.}

\section{Methods}

A retrospective analysis of 500 patients post-adenotonsillectomy was undertaken. The incidence of residual snoring, residual sleep symptoms and results of RAST, as well as total immunoglobulin $E$ (lgE) after surgical intervention, were documented.

\section{Results}

Children with positive RAST results or elevated total IgE had a significantly greater incidence of residual snoring post-adenotonsillectomy $(P=0.049)$ and residual sleep symptoms after surgery $(P<0.0001)$.

\section{Discussion}

A positive RAST or elevated $\lg E$ in children with SDB was associated with incomplete resolution of snoring and residual sleep symptoms after adenotonsillectomy. Thus, there should be raised suspicion of allergic rhinitis in this population.
SLEEP DISORDERED BREATHING (SDB)

describes conditions ranging from primary snoring to obstructive sleep apnoea $(\mathrm{OSA})^{1}$ and affects approximately $7 \%$ of the paediatric population. ${ }^{2}$ It is associated with clinically significant cardiovascular morbidity and impaired cognitive development. ${ }^{3-5}$

SDB is characterised by partial or complete periodic obstruction of the upper airway while sleeping. This is often coupled with snoring, oxyhaemoglobin desaturation, repeated arousals and carbon dioxide retention. ${ }^{6}$

One major treatable cause of SDB is adenotonsillar hypertrophy. ${ }^{1}$ Thus, the recommended surgical management of SDB in this age group is adenotonsillectomy, which results in resolution of signs and symptoms in $80-90 \%$ of cases. ${ }^{7,8}$ In the remaining $10-20 \%$ of cases, among other factors such as ethnicity, obesity, sleep-state dependent laryngomalacia, craniofacial and congenital abnormalities, allergic rhinitis may also contribute to ongoing symptoms postoperatively. ${ }^{9}$

Allergic rhinitis results in nasal obstruction due to mucosal oedema and secretions. ${ }^{6}$ This increases upper airway resistance, predisposing children to partial or complete upper airway obstruction during sleep. ${ }^{10}$ Importantly, it may be a treatable component that contributes to residual symptoms such as snoring.

The aim of our study was to evaluate the largest cohort of children who have had adenotonsillectomy for SDB to determine if there is an association between radioallergosorbent testing (RAST) or elevated immunoglobulin E (IgE) levewith residual sleep symptoms in children.

\section{Methods}

The study analysed a retrospective cohort of 500 consecutive patients aged 1-16 years. An audit was conducted of children aged 0-12 years with sleep symptoms who underwent adenotonsillectomy and revealed features of allergic rhinitis on clinical examination or medical history from 2007 until the present. Those who underwent adenotonsillectomy for SDB by a single surgeon in the Illawarra region of New South Wales, Australia between July 2008 and September 2015 were reviewed. Patients were eligible for analysis if they were aged 1-16 years and had undergone adenotonsillectomy not in conjunction with any other procedure, had a serum RAST or IgE test performed intraoperatively and had not been previously diagnosed or treated for allergic rhinitis. The indication for RAST was determined by a personal or family history of allergic rhinitis, asthma or eczema, or examination findings suggestive of allergic rhinitis; this information was recorded for each patient by the operating surgeon prior to intervention. Follow-up of at least six weeks was necessary to be eligible for assessment. Of the 500 patients, 28 (5.6\%) were lost to follow-up, leaving 472 available for complete analysis.

Patients were asked questions pertaining to symptoms considered to be indicative of allergic rhinitis, including persistent sneezing, persistent itching or rubbing of the nose, rhinorrhoea and nasal obstruction. ${ }^{11}$ Examination findings considered to be indicative of allergic rhinitis included prominent inferior turbinates, allergic shiners, allergic dorsal 
nasal skin creases from persistent rubbing of the nose and mucoid stranding on anterior rhinoscopy. ${ }^{11}$ For each case, the history and physical examination findings were noted at the initial assessment and six weeks postoperatively by the surgeon who had performed the procedure.

Other residual symptoms (ORS) besides snoring were determined by collateral history from carers and specifically recorded pre-operatively and six weeks after surgery. Carers were asked about frequent waking, restless sleep, witnessed gasping or choking, mouth-breathing, frequent misbehaviour (defined as frequent misbehaviour raising parental or teacher concerns with a variation of behaviour outside of subjective norms including tantrums, being disruptive, disrespectful or attention seeking), daytime somnolence, daytime naps, poor concentration, sleep walking, night terrors, bruxism and nocturnal enuresis.

When assessing patients, all preoperative and post-operative assessments and questions were asked by the surgeon performing the procedure. Although a standardised questionnaire was not used, specific questions were asked to determine if significant elements of the history, as explained above, were present or absent, the significance of which has been previously described in the literature. ${ }^{11}$ Similarly, the same physical examination, observing for specific signs as previously decribed, ${ }^{11}$ was undertaken by the same surgeon. To minimise the risk of bias, data were collected and assessed independent of the surgeon involved in the care of patients.

\section{Radioallergosorbent testing analysis}

Biochemical analysis of allergic rhinitis was performed using the standard RAST technique as previously described, ${ }^{12}$ and serum IgE levels were detected using ImmunoCAP Total IgE Fluoroenzyme immunoassay (Thermo Fisher Scientific Inc). All 500 patients underwent RAST and IgE testing by obtaining serum vials at the onset of anaesthesia, prior to commencing the procedure. The RAST analysis was used to detect atopic disease to inhalant allergens (house dust mite, grass pollen, mould, animal mix) as well as staple food mix.
The null hypothesis of no association between factors (RAST, allergic rhinitis signs or symptoms or allergens) and outcomes of residual snoring and other ORS was assessed with a Fisher's exact test of independence (or no association). Two-way tables were generated with the presence or absence of a factor against no residual snoring and residual snoring. A Fisher's exact $P$ value $<0.05$ was declared significant. The proportions of patients with the outcomes of interest for each level of the factor were estimated and converted to percentages for ease of interpretation. Exact Clopper-Pearson confidence intervals (CI) for binomial proportions were estimated for each proportion and converted to percentages. Differences between these percentages were estimated with the corresponding 95\% exact unconditional confidence limits for the difference. Ethics approval was gained from the University of Wollongong Human Research Ethics Committee: HE16/155 UOW HREC.

\section{Results}

\section{Allergic rhinitis and its association with other residual symptoms and snoring}

The percentage of children with a positive RAST or elevated IgE in our cohort was $37.3 \%$ (176/472). The majority of patients who underwent adenotonsillectomy for SDB had resolution of snoring $(92.4 \%$, $436 / 472) ; 79.8 \%(348 / 436)$ of these patients had no ORS (Figure 1). A total of $7.6 \%(36 / 472)$ had residual snoring, and $20.2 \%(88 / 436)$ had ORS at the sixweek follow-up. Of these residual snorers, $55.6 \%(20 / 36)$ were positive on RAST/ IgE testing. In patients with ORS but no snoring, 67.0\% (59/88) were positive on RAST/IgE testing (Figure 1).

To determine if there was a relationship between RAST/IgE status and residual snoring or ORS, a Fisher's exact test was performed. A significant association was found between a positive RAST/ IgE test and residual snoring $(P=0.049)$. Approximately $11 \%$ of patients with a positive RAST/IgE test had residual snoring, compared with $5.5 \%$ of those with a negative RAST/IgE test, an increase of $5.4 \%$ (95\% CI: 0.16, 10.6; Table 1). Furthermore, an association was identified between patients who tested positive for RAST/IgE and ORS post-surgery $(P<0.0001$; Table 2$)$, with approximately $38.8 \%$ of patients with ORS, compared with approximately $13.8 \%$ without ORS (difference $=25 \%, 95 \%$ CI: 16.0, 33.10). Therefore, a positive RAST/IgE result is associated with ORS post-adenotonsillectomy and may also be associated with residual snoring.

It is unclear whether allergic rhinitis signs and symptoms alone are able to predict residual snoring or ORS after surgery. To determine this, we performed Fisher's exact tests between residual snoring and the variable of interest (allergic rhinitis signs and symptoms). We found no significant relationship between the presence of allergic rhinitis signs or symptoms prior to surgery and residual snoring $(P=0.43)$. Of those patients who had ongoing residual snoring after surgery, approximately $10.1 \%$ of patients had allergic rhinitis signs or symptoms prior to surgery, compared with $7.3 \%$ who did not present with allergic rhinitis signs or symptoms (Table 1 ).

The same test was performed to determine if allergic rhinitis signs or symptoms were associated with ORS. There was no significant difference between those patients who presented with allergic rhinitis signs and symptoms prior to surgery and those who had ongoing ORS post-surgery (28.8\% versus $22.7 \%, P=0.27$; Table 2 ). This suggests that RAST/IgE testing may help to predict snoring or ORS post-surgery and that signs or symptoms of allergic rhinitis prior to surgery are not a sufficient predictor alone.

\section{Common allergens}

An analysis was conducted to determine whether there was a relationship between common allergens and residual snoring or ORS. Dust mites $(28.2 \%, 141 / 500)$ and grass pollen $(7.8 \%, 39 / 500)$ were the most common allergens. There was a significant association between house dust mite allergy and the presence of residual snoring, with approximately $12.1 \%$ of snorers testing positive for house dust mite allergy, compared with $5.7 \%$ of snorers 
who did not have a positive test (difference $=6.4 \%, 95 \%$ CI: $0.4,12.3, P=0.03)$. No significant relationship was found for other common allergens (data not shown).

Patients who had ongoing ORS postsurgery also had a significantly higher incidence of allergy to dust mites, compared with those who had a negative RAST/IgE result for the allergen $(39.7 \%$ versus $16.6 \%$ respectively, difference $=$ $23.1 \%$, 95\% CI: 13.3 , 32.6, $P<0.0001)$. The same relationship was also seen for those who were positive for grass pollen (41\% versus $21.9 \%$, difference $=19.1 \%$, 95\% CI: 2.7, 35.3, P= 0.01) and mould (64.3\% versus $22.3 \%$, difference $=42 \%$, 95\% CI: $15,69.3, P=0.001)$ but not to staple food mix (34.5\% versus $22.8 \%$, $P=0.17)$ or animal dander $(28.6 \%$ versus $23.2 \%, P=0.5)$, although the number of patients with an allergy to the latter three allergens was limited (Table 3).

\section{Gender}

Overall, we found no significant difference between the proportion of males and females who had positive RAST/IgE results (41.2\% versus $33.9 \%, P=0.12)$. Furthermore, a three-way assessment of residual snoring state by gender by RAST/IgE status revealed no significant difference between males and females that were RAST-positive and presented with or without residual snoring (data not shown).

\section{Discussion}

This is the largest cohort study to evaluate the incidence of allergic rhinitis and residual symptoms postadenotonsillectomy in the Australian paediatric population. The majority of patients experienced SDB resolution irrespective of RAST or IgE status, a finding supported by previous literature. ${ }^{13}$ However, our results suggest an association with a higher incidence of residual snoring or ORS when positive for RAST/IgE. Although a possible advantage of pre-operative RAST or IgE testing for this population would be to determine a positive status and ensure closer follow-up after surgery, given the greater likelihood of resolution, it may be more feasible to perform RAST or IgE testing in those children with ongoing snoring or ORS.

Our results support previous research with smaller cohorts highlighting a greater incidence of incomplete resolution of SDB symptoms in children who had positive RAST or IgE results. ${ }^{1,7}$ However, we did not find a significant relationship with residual snoring or ORS in patients with allergic rhinitis signs and symptoms irrespective of RAST or IgE status. Therefore, history and examination findings of allergic rhinitis alone may be inadequate in guiding the physician to suspect a greater potential for residual snoring or ORS to be present postoperatively without using a RAST or IgE test.

Our results are comparable to previous papers of smaller cohorts with respect to incidence of patients with positive RAST and snoring. ${ }^{13,14}$ The postulated cause of SDB in patients with allergic rhinitis may be related to prolonged oral

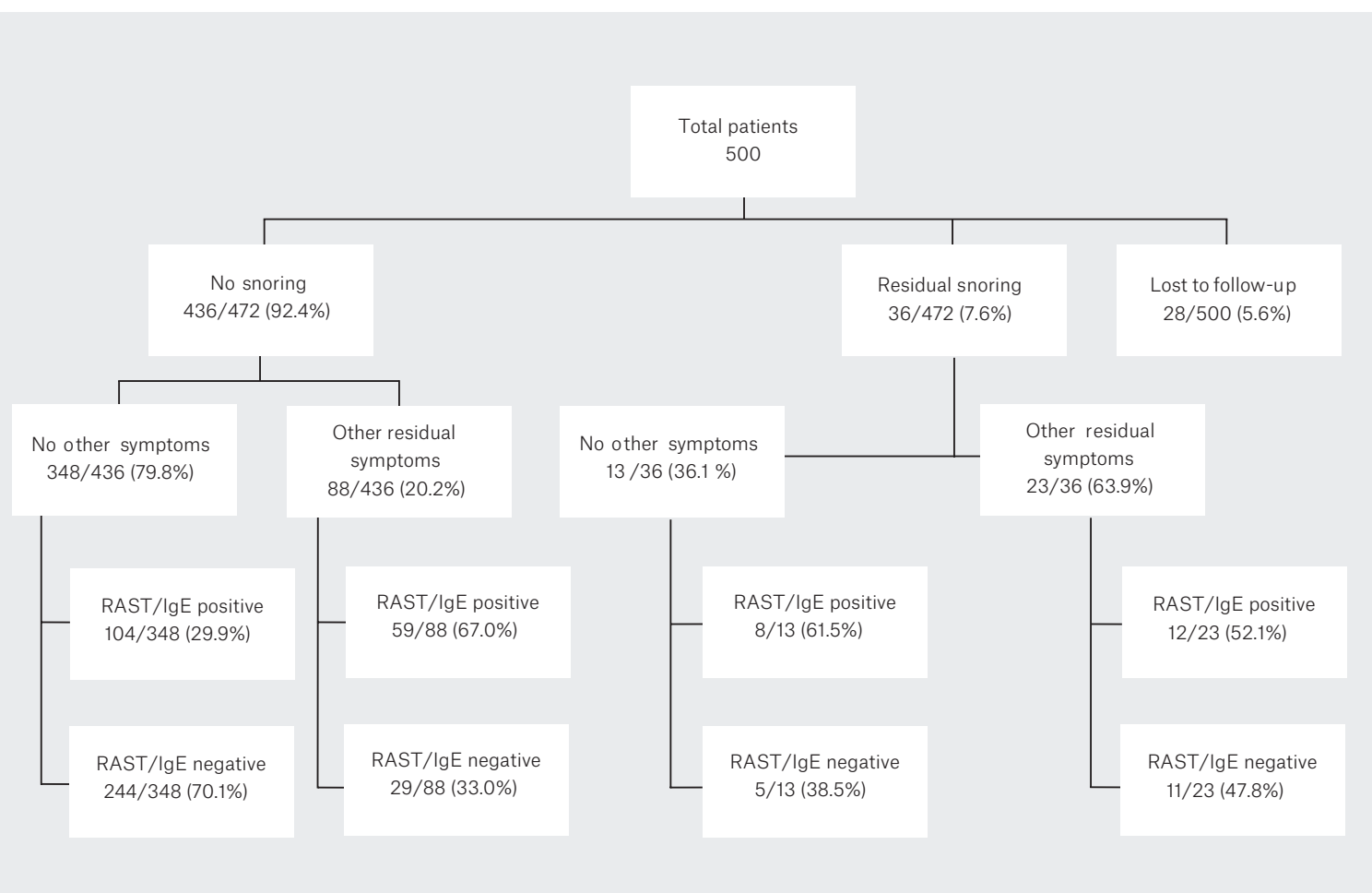

Figure 1. Flow chart showing the number of patients with and without snoring and other residual symptoms expressed as a ratio and as a percentage 
breathing resulting in caudal mandibular growth ${ }^{15}$ and oedematous nasal mucosa causing turbulent airflow as well as nasal obstruction, both of which may be present as a result of an IgE-mediated immune response to an aeroallergens in patients with allergic rhinitis. This nasal obstruction can result in persistent mouthbreathing and may be a major contributing factor to SDB. ${ }^{16}$ Allergic rhinitis may also contribute to SDB via other common symptoms including nocturnal pruritus, nasal itching and rhinorrhea. ${ }^{16}$ Thus, establishing RAST positivity may be useful to predict patients at greater risk of ORS or residual snoring post-adenotonsillectomy. However, further prospective studies are required to investigate this.
Previous smaller studies have suggested improvement in SDB in children with active allergic rhinitis treatment. ${ }^{17}$ Our data suggest a significantly higher incidence of ORS in those patients who had a positive RAST or IgE test, but the management of allergic rhinitis is beyond the scope of this paper. However, on the basis of these results, avoiding exposure

Table 1. Numbers of subjects in each cross-classification of residual snoring and variables of interest

\begin{tabular}{|c|c|c|c|c|c|c|c|c|}
\hline \multirow[b]{2}{*}{ Variable } & \multirow[b]{2}{*}{ Measure } & \multirow[b]{2}{*}{ No } & \multicolumn{3}{|c|}{ Residual snoring } & \multirow[b]{2}{*}{$95 \% \mathrm{Cl}$} & \multicolumn{2}{|c|}{$\begin{array}{l}\text { Difference (positive/present } \\
\text { to negative/absent) }\end{array}$} \\
\hline & & & Yes & Total & Percentage (SE) & & $\begin{array}{l}\text { Proportion } \\
(95 \% \mathrm{Cl})\end{array}$ & $P$ \\
\hline \multirow[t]{3}{*}{ RAST/IgE } & Negative & 273 & 16 & 289 & $5.5( \pm 1.4)$ & $2.9,8.8$ & & \\
\hline & Positive & 163 & 20 & 183 & $10.9( \pm 2.3)$ & $6.4,5.5$ & & \\
\hline & Difference & & & & & & $5.4(0.2,10.6)$ & 0.049 \\
\hline \multirow[t]{2}{*}{$\begin{array}{l}\text { Allergic rhinitis } \\
\text { signs or symptoms }\end{array}$} & Absent & 384 & 30 & 414 & $7.3( \pm 1.3)$ & $4.8,9.7$ & & \\
\hline & Present & 52 & 6 & 58 & $10.1( \pm 3.9)$ & 2,8 & & \\
\hline
\end{tabular}

Table 2. Numbers of subjects in each cross-classification of other residual symptoms (ORS) and variables of interest

\begin{tabular}{|c|c|c|c|c|c|c|c|c|}
\hline \multirow[b]{2}{*}{ Variable } & \multirow[b]{2}{*}{ Measure } & \multicolumn{5}{|c|}{ Other residual symptoms (ORS) } & \multicolumn{2}{|c|}{$\begin{array}{l}\text { Difference (positive/present } \\
\text { to negative/absent) }\end{array}$} \\
\hline & & No & Yes & Total & Proportion (SE) & $95 \% \mathrm{Cl}$ & $\begin{array}{l}\text { Proportion } \\
(95 \% \mathrm{Cl})\end{array}$ & $P$ \\
\hline \multirow[t]{3}{*}{ RAST/IgE } & Negative & 249 & 40 & 289 & $13.8( \pm 2.0)$ & $9,17.8$ & & \\
\hline & Positive & 112 & 71 & 183 & $38.8( \pm 3.6)$ & $31.7,46.3$ & & \\
\hline & Difference & & & & & & $25(16.9,33.1)$ & $<0.0001$ \\
\hline \multirow[t]{2}{*}{$\begin{array}{l}\text { Allergic rhinitis } \\
\text { signs or symptoms }\end{array}$} & Absent & 320 & 94 & 414 & $22.7( \pm 2.1)$ & $18.7,26.7$ & & \\
\hline & Present & 41 & 17 & 58 & $28.8( \pm 5.9)$ & $17.2,40.3$ & & \\
\hline
\end{tabular}

Cl, confidence interval; IgE, immunoglobulin E; RAST, radioallergosorbent test; SE, standard error 
to allergens may be of some benefit if detection can be specified. Allergens detected to have a statistically significant association with residual snoring and ORS in our cohort were dust mite and grass pollens. Along with avoidance of such allergens, topical or systemic methods of medical management may be of benefit, although further prospective research is required to determine treatment effects.

With respect to gender, previous literature has suggested that males are more likely to have SDB than females. ${ }^{18}$ Allergic rhinitis is also more prevalent in males, ${ }^{19,20}$ which raises the question of whether male patients are more likely to have ongoing symptoms postadenotonsillectomy than females. In our cohort, there was no significant difference between gender and the incidence of allergic rhinitis detected clinically or via RAST/IgE. Similarly, there was no difference in the incidence of residual snoring or ORS when comparing male and female patients. Thus, gender may not predispose patients to a greater risk of residual snoring or ORS post adenotonsillectomy.

Our study was a single-arm retrospective analysis with the aim of identifying the incidence of residual snoring or ORS in children with SDB and its association with allergic rhinitis post-adenotonsillectomy. Thus, there are limitations to our study. Questions regarding symptoms were uniformly asked of all carers for patients; however, standardised questionnaires may have been better in assessing recurrence and severity of sleep symptoms.

The data were not controlled for confounding contributors to ongoing SDB, such as obesity, ${ }^{21}$ and follow-up was at a single time frame of six weeks postoperatively. Subsequent review

Table 3. Numbers of subjects in each cross-classification of other residual symptoms and variables of interest

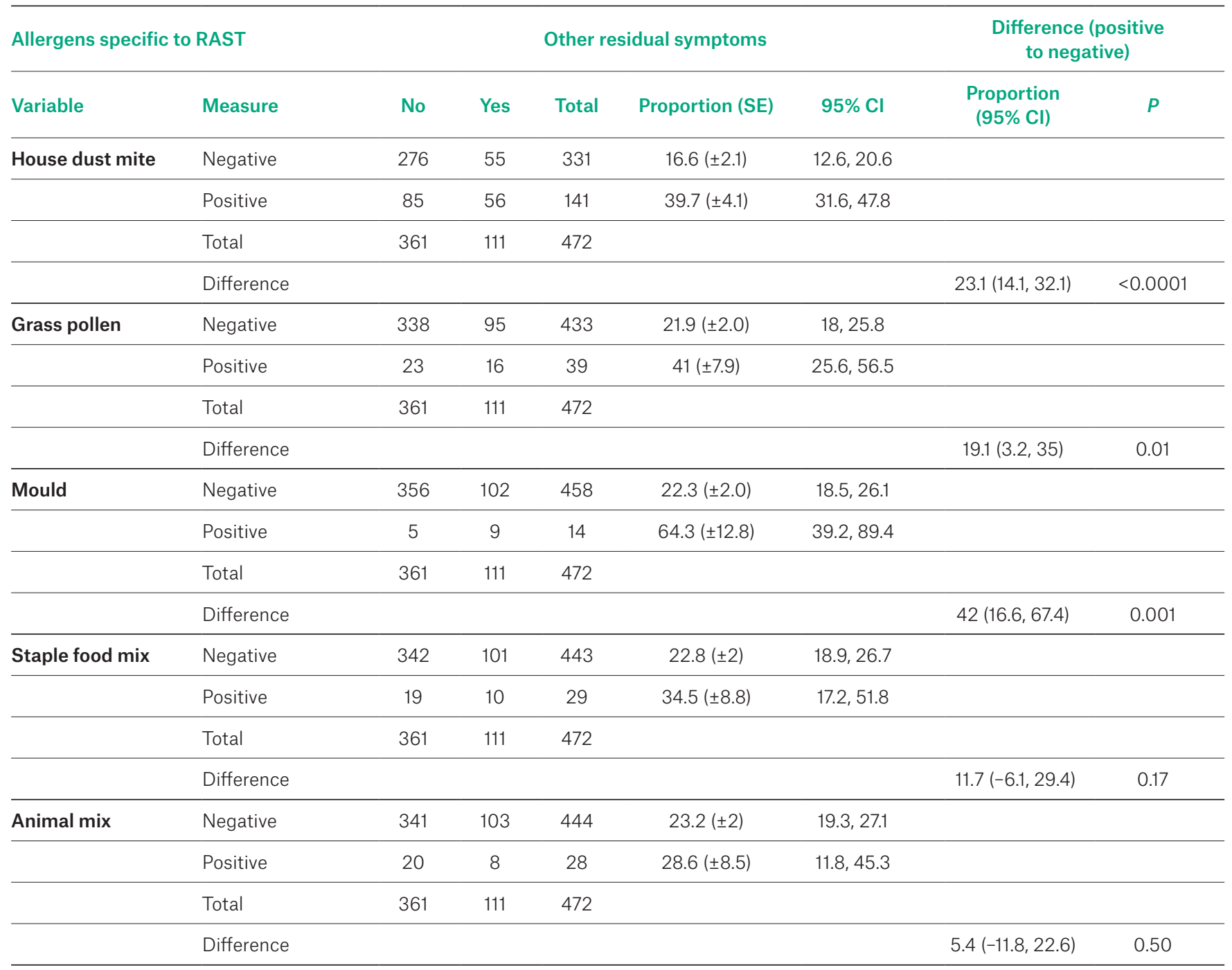

Cl, confidence intervals; RAST, radioallergosorbent test; SE, standard error 
thereafter may have been warranted to determine persistence of ORS. In terms of age, our cohort was restricted to patients aged 1-16 years; however, this does leave considerable variation and must be considered as a potential confounder in our review. Furthermore, it is important to note that a positive RAST or IgE result may not be as significant a predictor as other variables not assessed in this study. However, the association remains interesting.

\section{Conclusion}

A positive RAST or IgE testing result is associated with incomplete resolution of snoring or ORS after adenotonsillectomy in children, possibly predisposing them to a more reactive airway and disrupted sleep. Our current data suggest that RAST/ IgE testing in children with SDB may be a relatively simple and cost-effective diagnostic adjunct to identify those patients presenting to practitioners after surgery with residual sleep symptoms that may be amenable to allergy treatments. Prospective research in a consecutive cohort of children undergoing adenotonsillectomy for SDB has commenced, to determine if a positive RAST/IgE result can predict those patients who are more likely to have residual snoring and ORS after this procedure.

\section{Implications for general practice}

- Allergic rhinitis diagnosed via RAST or IgE is associated with residual snoring and ORS in children with SDB.

- RAST/IgE testing is relatively easy and inexpensive to perform. In conjunction with a focused history and examination, the test may allow the primary care physician to decide on treatment for allergy where there are residual symptoms of SDB.

\section{Authors}

Lernik Sarkissian BAppScExSS, MPhty, MBBS, Otolaryngology, Head \& Neck registrar, Wollongong Hospital; Illawarra ENT Head and Neck Clinic Wollongong, NSW. Is095@uowmail.edu.au Lala Sarkissian BMedSc (Hons), Medical student, The University of Notre Dame, Sydney, NSW

Thomas E Stewart BMedSc, MBBS, MMed, Otolaryngology, Head \& Neck Fellow, Wollongong Hospital, Sydney, NSW
Thomas Guy PHD, Medical student, Wollongong Hospital, Wollongong, NSW

Stuart G Mackay BScMed, MBBS(Hons), Professor in Otolaryngology, Head \& Neck Surgery, Wollongong Hospital; Illawarra ENT Head and Neck Clinic; The Woolcock Institute of Medical Research, Wollongong, NSW

Competing interests: None.

Provenance and peer review: Not commissioned, externally peer reviewed.

\section{Acknowledgements}

We would like to thank Dr Caro Badcock at The Woolcock Institute of Medical Research for her assistance with performing the statistical analysis of this research article.

\section{References}

1. Anuntaseree W, Rookkapan K, Kuasirikul S, Thongsuksai P. Snoring and obstructive sleep apnea in Thai school-age children: Prevalence and predisposing factors. Pediatr Pulmonol 2001;32(3):22-27.

2. Lumeng JC, Chervin RD. Epidemiology of pediatric obstructive sleep apnea. Proc Am Thorac Soc 2008:5(2):242-52. doi: 10.1513/pats.200708$135 \mathrm{MG}$

3. Amin R, Somers VK, McConnell K, et al. Activityadjusted 24-hour ambulatory blood pressure and cardiac remodeling in children with sleep disordered breathing. Hypertension 2008;51(1):8491. Epub 2007 Dec 10

4. Gozal D, Kheirandish-Gozal L, Serpero LD, Sans Capdevila O, Dayyat E. Obstructive sleep apnea and endothelial function in school-aged nonobese children: Effect of adenotonsillectomy. Circulation 2007;116(20):2307-14

5. Tsaoussoglou M, Bixler EO, Calhoun S Chrousos GP, Sauder K, Vgontzas AN. Sleepdisordered breathing in obese children is associated with prevalent excessive daytime sleepiness, inflammation, and metabolic abnormalities. J Clin Endocrinol Metab 2010;95(1):143-50. doi: 10.1210/jc.2009-0435.

6. Bhattacharjee R, Kheirandish-Gozal L, Spruyt $\mathrm{K}$, et al. Adenotonsillectomy outcomes in treatment of obstructive sleep apnea in children: A multicenter retrospective study. Am J Respir Crit Care Med 2010;182(5):676-83. doi: 10.1164/ rccm.200912-19300C.

7. Kim DK, Han DH. Impact of allergic rhinitis on quality of life after adenotonsillectomy for pediatric sleep-disordered breathing. Int Forum Allergy Rhinol 2015:5(8):741-46. doi: 10.1002/ alr.21529.

8. Brietzke SE, Gallagher D. The effectiveness of tonsillectomy and adenoidectomy in the treatment of pediatric obstructive sleep apnea/hypopnea syndrome: A meta-analysis. Otolaryngol Head Neck Surg 2006;134(6):979-84.

9. Huang YS, Guilleminault $\mathrm{C}$, Lee LA, Lin $\mathrm{CH}$, Hwang FM. Treatment outcomes of adenotonsillectomy for children with obstructive sleep apnea: A prospective longitudinal study. Sleep 2014;37(1):71-76. doi: 10.5665/sleep.3310.

10. McColley SA, Carroll JL, Curtis S, Loughlin GM, Sampson HA. High prevalence of allergic sensitization in children with habitual snoring and obstructive sleep apnea. Chest 1997;111(1):170-73.

11. Bousquet J, Khaltaev N, Cruz AA, et al. Allergic Rhinitis and its Impact on Asthma (ARIA) 2008 update (in collaboration with the World Health
Organization, GA(2)LEN and AllerGen). Allergy 2008;63 Suppl 86:8-160. doi: 10.1111/j.13989995.2007.01620.x.

12. Ng DK, Chan $\mathrm{CH}$, Hwang GY, Chow PY, Kwok KL. A review of the roles of allergic rhinitis in childhood obstructive sleep apnea syndrome. Allergy Asthma Proc 2006;27(3):240-42.

13. Schechter MS; Section on Pediatric Pulmonology, Subcommittee on Obstructive Sleep Apnea Syndrome. Technical report: Diagnosis and management of childhood obstructive sleep apnea syndrome. Pediatrics 2002;109(4):e69.

14. Morgenthaler TI, Kapen S, Lee-Chiong T, et al. Practice parameters for the medical therapy of obstructive sleep apnea. Sleep 2006;29(8):1031-35.

15. Craig TJ, McCann JL, Gurevich F, Davies MJ. The correlation between allergic rhinitis and sleep disturbance. J Allergy Clin Immunol 2004;114(5 Suppl):S139-45.

16. Wallace DV, Dykewicz MS, Bernstein DI, et al. The diagnosis and management of rhinitis: An updated practice parameter. J Allergy Clin Immunol 2008;122(2 Suppl):S1-84. doi: 10.1016/j.jaci.2008.06.003.

17. Mansfield LE, Diaz G, Posey CR, Flores-Neder J. Sleep disordered breathing and daytime quality of life in children with allergic rhinitis during treatment with intranasal budesonide. Ann Allergy Asthma Immunol 2004;92(2):240-44.

18. Chau KW, Ng KK, Kwok KL, Cheung MY. Survey of children with obstructive sleep apnea syndrome in Hong Kong of China. Chin Med J (Engl) 2004;117(5):657-60.

19. Poachanukoon O, Kitcharoensakkul M. Snoring and sleep problems in children with and without allergic rhinitis: A case control study. J Med Assoc Thai 2015;98 Suppl 2:S138-44.

20. Salehi M, Bakhshaee M, Ashtiani SJ, Najafi M Sehatbakhsh S, Hossainzadeh M. Parental smoking and allergic rhinitis in children. Int Forum Allergy Rhinol 2014;4(5):357-60. doi: 10.1002/ alr.21286.

21. Li AM, Zhu Y, Au CT, Lee DLY, Ho C, Wing YK Natural history of primary snoring in schoolaged children: A 4-year follow-up study. Chest 2013;143(3):729-35. doi: 10.1378/chest.12-1224. 